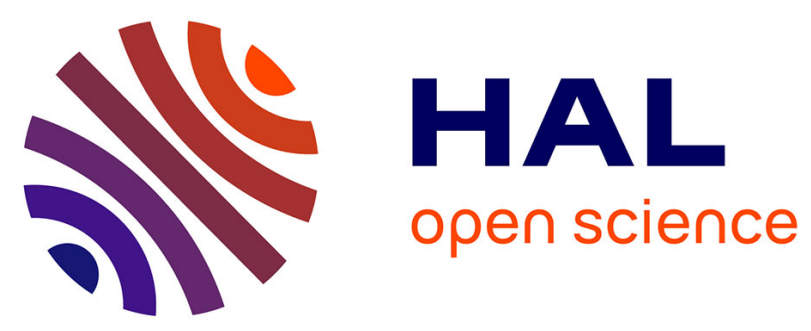

\title{
Long range gravity tests and the Pioneer anomaly
}

Serge Reynaud, Marc-Thierry Jaekel

\section{To cite this version:}

Serge Reynaud, Marc-Thierry Jaekel. Long range gravity tests and the Pioneer anomaly. International Journal of Modern Physics D, 2007, 16, pp.2091 - 2105. 10.1142/S0218271807011656 . hal-00110783

\section{HAL Id: hal-00110783 \\ https://hal.science/hal-00110783}

Submitted on 31 Oct 2006

HAL is a multi-disciplinary open access archive for the deposit and dissemination of scientific research documents, whether they are published or not. The documents may come from teaching and research institutions in France or abroad, or from public or private research centers.
L'archive ouverte pluridisciplinaire HAL, est destinée au dépôt et à la diffusion de documents scientifiques de niveau recherche, publiés ou non, émanant des établissements d'enseignement et de recherche français ou étrangers, des laboratoires publics ou privés. 


\title{
LONG RANGE GRAVITY TESTS AND THE PIONEER ANOMALY
}

\author{
Serge Reynaud ${ }^{1}$ and Marc-Thierry Jaekel $^{2}$ \\ ${ }^{1}$ Laboratoire Kastler Brossel*, Université Pierre et Marie Curie, case 74, \\ Campus Jussieu, F75252 Paris cedex 05 \\ reynaud@spectro.jussieu.fr \\ ${ }^{2}$ Laboratoire de Physique Théorique ${ }^{\dagger}$, Ecole Normale Supérieure, \\ 24 rue Lhomond, F75231 Paris cedex 05 \\ jaekel@lpt.ens.fr
}

\begin{abstract}
Experimental tests of gravity performed in the solar system show a good agreement with general relativity. The latter is however challenged by the Pioneer anomaly which might be pointing at some modification of gravity law at ranges of the order of the size of the solar system. As this question could be related to the puzzles of "dark matter" or "dark energy", it is important to test it with care. There exist metric extensions of general relativity which preserve the well verified equivalence principle while possibly changing the metric solution in the solar system. Such extensions have the capability to preserve compatibility with existing gravity tests while opening free space for the Pioneer anomaly. They constitute arguments for new mission designs and new space technologies as well as for having a new look at data of already performed experiments.
\end{abstract}

\section{INTRODUCTION}

The commonly heard assertion that gravity tests show a good agreement with general relativity (GR) has to be understood as a set of more detailed statements [1, 2]. It first implies that the gravitational field may be identified with the metric tensor $g_{\mu \nu}$ in a Riemannian space-time, as a consequence of the fact that the equivalence principle is one of the most accurately verified properties of nature. It then means that this metric tensor appears to have a form close to that predicted by GR, as shown by the confrontations of observations with the family of more general PPN solutions. This second statement can be put under the alternative form of a good agreement of the gravity force law with the prediction of GR, deviations being predicted by unification models but not observed to date [3, 幽.

Besides these successes, GR is challenged by observations performed at various scales. First, anomalies are known to affect the rotation curves of galaxies. They are commonly accounted for by introducing "dark matter" to reproduce these curves [5, 6]. Further anomalies have been detected more recently in the relation between redshifts and luminosities, showing an acceleration of cosmic expansion. They are usually interpreted as due to the presence of some "dark energy" [7, 8, Both components of the "dark side" of the universe have no known origin and are not observed through other means than the gravitational anomalies they have been designed to cure. As long as this situation is lasting, the related anomalies may as well be interpreted as long range deviations from GR 9, 10, 11].

The Pioneer anomaly constitutes a new piece of information in this puzzling context, which might already

${ }^{*}$ CNRS, ENS, UPMC reveal an anomalous behaviour of gravity at scales of the order of the size of the solar system [12, 13]. Though a number of mechanisms have been considered to this aim 14, 15, 16, 17, 18, 19, the anomaly has escaped up to now all attempts of explanation as a systematic effect generated by the spacecraft itself or its environment. The importance of the Pioneer anomaly for space navigation already justifies it to be submitted to further scrutiny. Meanwhile its potential impact on fundamental physics cannot be underestimated, since the possibility exists that the Pioneer anomaly be the first hint of a long range modification of gravity law 20, 21, 22, 23, 24, 25, 26.

These questions are reviewed in the present paper, with the emphasis put on the key issue of the compatibility of the Pioneer anomaly with other gravity tests.

\section{GRAVITY TESTS IN THE SOLAR SYSTEM}

General Relativity (GR) provides us with an excellent description of gravitational phenomena in the solar system. In order to discuss the meaning of this common statement, we first recall the basic features of this theoretical description and then briefly review the experimental evidences supporting it.

In order to apply the principle of relativity to accelerated motions, Einstein introduced what is now called the equivalence principle 27, 28. A weak form of this principle is expressed by the universality of free fall, a central property of the theory of gravitation since Galileo and Newton which acquires with Einstein a geometrical significance, gravitation fields being identified with the metric tensor $g_{\mu \nu}$ in a Riemannian space-time. Ideal atomic clocks measure the proper time $\int d s$ along their trajectory in space-time with $d s^{2} \equiv g_{\mu \nu} d x^{\mu} d x^{\nu}$. Meanwhile freely falling motions are the geodesics of this Riemannian space-time, that is also the curves which extremize the integral $\int d s$.

The equivalence principle is one of the best ever tested 
properties of nature. Potential violations are usually parametrized by a relative difference $\eta$ in the accelerations $a_{1}$ and $a_{2}$ undergone by two test bodies of different compositions in free fall at the same location and with the same velocity. Modern experiments constrain the parameter $\eta$ to stay below the $10^{-12}$ level. These experiments test the principle at distances ranging from the millimeter in laboratory experiments (Ref. 溲 and references in) to the sizes of Earth-Moon [29] or Sun-Mars orbit [30].

The geometrical interpretation is the very core of GR, but it is not sufficient to fix the latter theory. In order to do that, it is necessary to write also the equations determining the metric tensor from the distribution of energy and momentum in space-time or, in other words, to fix the form of the coupling between curvature and stress tensors. Among the curvature tensors available in Riemannian geometry, the Einstein tensor $E_{\mu \nu} \equiv R_{\mu \nu}-\frac{1}{2} g_{\mu \nu} R$ is defined from the Ricci tensor $R_{\mu \nu}$ and scalar curvature $R$ so that it has a null covariant divergence $D^{\mu} E_{\mu \nu} \equiv 0$. This geometrical property has to be compared with the physical property $D^{\mu} T_{\mu \nu} \equiv 0$ which expresses conservation of energy and momentum as the condition of null divergence of the stress tensor $T_{\mu \nu}$. Note that the latter relation is a necessary and sufficient condition for motions of test masses to follow geodesics.

GR corresponds to a simple proportionality relation between the two tensors $E_{\mu \nu}$ and $T_{\mu \nu}$, the constant being determined from the Newton gravitation constant $G_{N}$ and the velocity of light $c$,

$$
E_{\mu \nu}=\frac{8 \pi G_{N}}{c^{4}} T_{\mu \nu}
$$

This Einstein-Hilbert equation [31, 32, 33] is tested through comparisons of its predictions with observations or experiments. To this aim, the metric tensor in the solar system is first deduced by solving (1). In the simple case where the gravity source, i.e. the Sun, is described as a point-like motion-less mass $M$, the metric can be written as an expansion in terms of the Newton potential $\phi$

$$
\begin{aligned}
d s^{2} & =g_{00} c^{2} d t^{2}+g_{r r}\left(d r^{2}+r^{2}\left(d \theta^{2}+\sin ^{2} \theta d \varphi^{2}\right)\right) \\
g_{00} & =1+2 \phi+2 \phi^{2}+\ldots \quad, \quad g_{r r}=-1+2 \phi+\ldots \\
\phi & \equiv-\frac{\kappa}{r} \quad, \quad \kappa \equiv \frac{G_{N} M}{c^{2}} \quad, \quad|\phi| \ll 1
\end{aligned}
$$

Spherical coordinates have been used ( $t$ and $r$ are time and radius, $\theta$ and $\varphi$ colatitude and azimuth angles) with the Eddington gauge convention of isotropic spatial coordinates. $\kappa$ is the gravitational radius of the Sun $\sim 1.5 \mathrm{~km}$.

GR is usually tested through its confrontation with the enlarged family of parametrized post-Newtonian (PPN) metric tensors introduced by Eddington 34 and then developed by several physicists [35, 36, 37, 38]

$$
g_{00}=1+2 \alpha \phi+2 \beta \phi^{2}+\ldots \quad, \quad g_{r r}=-1+2 \gamma \phi+\ldots
$$

The three parameters $\alpha, \beta$ and $\gamma$ are constants, the first of which can be set to unity by fixing Newton constant
$G_{N}$. Within the PPN family, GR thus corresponds to $\gamma=\beta=1$. Anomalies $\gamma-1$ or $\beta-1$ of these Eddington parameters affect motions, i.e. the geodesics associated with the metric (3), and they can therefore be measured by comparing observations with predictions.

Experiments now performed for more than four decades have led to more and more constraining bounds on these anomalies. For example, Doppler ranging on Viking probes in the vicinity of Mars 30 and deflection measurements using VLBI astrometry 39 or radar ranging on the Cassini probe [40] have given smaller and smaller values of $|\gamma-1|$, with presently a bound of a few $10^{-5}$. Analysis of the precession of planet perihelions 41 and of the polarization by the Sun of the Moon orbit around the Earth [42] have led to determinations of linear superpositions of $\beta$ and $\gamma$, resulting now to $|\beta-1|$ smaller than a few $10^{-4}$.

An alternative manner to test GR has been to check the $r$-dependence of the Newton potential, that is also of the component $g_{00}$ in (3). Hypothetical modifications of its standard expression, predicted by unification models, are usually parametrized in terms of an additional Yukawa potential depending on two parameters, the range $\lambda$ and the amplitude $\alpha$ measured with respect to Newton potential [3]. The presence of such a Yukawa correction has been looked for on a large range of distances. The accuracy of short range tests has been recently improved, as gravity experiments were pushed to smaller distances 43, 44, 45 and as Casimir forces, which become dominant at submillimeter range, were more satisfactorily taken into account 46, 47, 48, 49. On the other side of the distance range, long range tests of the Newton law are performed by following the motions of planets or probes in the solar system. They also show an agreement with GR with a good accuracy for ranges of the order of the Earth-Moon 29] or Sun-Mars distances [50, 51, 52,. When the whole set of results is reported on a global figure (see Fig. 1 in Ref. [53] reproduced thanks to a courtesy of Coy et al [54]), it appears that windows remain open for violations of the standard form of Newton force law at short ranges, below the millimeter, as well as long ones, of the order of or larger than the size of the solar system.

To sum up this discussion, tests of gravity confirm its metric interpretation and provide strong evidence in favor of gravitation theory being very close to GR. A few exceptions exist, among which notably the anomalous observations recorded on Pioneer probes. We will see below that this contradiction between Pioneer observations and other gravity tests may be cured in an extended framework, where deviations from GR may show a scale dependence. It is precisely the merit of Newton force law tests to shed light on this possibility of a scale dependence, with any specific experiment being only sensitive to a given range of distances. The issue of scale dependence has to be considered with great attention, especially in the context recalled in the Introduction where questions arise about the validity of GR at galactic or cosmic scales. 
As recalled in forthcoming sections, scale dependence is also a natural consequence of radiative corrections to GR to be taken into account.

\section{THE PIONEER ANOMALY}

After the discussions of the previous section, it is clear that the gravity laws have to be tested at all possible scales. It is of particular interest to study the largest scales attainable by man made instruments, in an attempt to bridge the gap between experiments made on Earth or in its vicinity and the much larger galactic and cosmic scales. The best example of such a strategy to this date is the NASA decision to extend Pioneer 10 \& 11 missions after their primary periods with the aim, among others, to test the laws of gravity at large heliocentric distances 55, 56. When considered as a gravity test, the extended Pioneer missions were the largest scaled test ever carried out, and they failed to confirm the known laws of gravity.

The anomaly was recorded on deep space navigation (DSN) tracking data from the Pioneer $10 \& 11$ probes 57. An up-link radio signal is emitted from Earth at a DSN station, it is then received and sent back by the probe, and the down-link radio signal is finally received on Earth at the same or another DSN station. For probes equipped with range measurement capabilities (which was not the case for Pioneer $10 \& 11$ ), the ranging observable is defined as half the time elapsed on Earth from the emission time to the reception time. For Pioneer $10 \& 11$ probes, the tracking technique was based on the measurement of the Doppler shift, a proper observable defined as the ratio of cycle counting rates of reference clocks located at emission and reception stations 58]. The same information can be encoded in a Doppler velocity $v$, with the ratio of received to emitted frequencies written as

$$
\frac{f}{f_{0}} \equiv \frac{1-\frac{v}{c}}{1+\frac{v}{c}}
$$

The observable $v$ represents a relative velocity of the probe with respect to the station, with relativistic and gravitational effects taken into account in the definition (4) and perturbations due to transmission media effects properly accounted for 13 .

These Doppler tracking data were analyzed during the travel of Pioneer $10 \& 11$ probes to the outer parts of the solar system. When the probes had reached a quieter environment, after flying by Jupiter and Saturn, a precise comparison of tracking data with predictions of GR showed that the observed Doppler velocity departed from the calculated Doppler velocity. The velocity was thus showing an anomaly $\delta v$ varying linearly with elapsed time (see the Fig. 8 of Ref. 13)

$$
\delta v \equiv v_{\text {observed }}-v_{\text {modelled }} \simeq-a_{P}\left(t-t_{\text {in }}\right)
$$

with $a_{P}$ an anomalous acceleration directed towards the Sun and having an approximately constant amplitude over a large range of heliocentric distances (AU $\equiv$ astronomical unit)

$$
a_{P}=(0.87 \pm 0.13) \mathrm{nm} \mathrm{s}^{-2} \quad, \quad 20 \mathrm{AU} \lesssim r_{P} \lesssim 70 \mathrm{AU}(6)
$$

It is worth emphasizing that the Pioneer anomaly has been registered on the two deep space probes showing the best navigation accuracy. Other anomalous observations have been reported for Ulysses and Galileo probes, but they were not as reliable as for Pioneer probes [13]. For other probes like Voyager $1 \& 2$ and Cassini, the navigation accuracy was not sufficient. In other words, the Pioneer gravity test has been performed twice with identical probes on similar trajectories - but escape directions opposite in the solar system - and the same result. This is not an impressive statistics when we compare it to the large number of tests confirming GR. In particular, when the possibility of an artefact onboard the probe or in its environment is considered, this artefact could be the same on the two probes. However, no satisfactory explanation of this kind has been found to date, though intensive efforts have been devoted to this aim. The extensive analysis of Anderson et al [13], published after years of cross checks, has been confirmed by an independent analysis [59]. Such independent reanalyses of the data remain an important tool to confirm or infirm the existence of the anomaly and they now experience a revival thanks to recently recovered data covering the whole period of Pioneer $10 \& 11$ missions from their launch to the last data point $[60,61,62,63$.

\section{A KEY QUESTION: IS THE PIONEER ANOMALY COMPATIBLE WITH OTHER GRAVITY TESTS}

In this context, the question of the compatibility of the observed Pioneer anomaly with other gravity tests acquires the status of a key issue. If there exist gravity theories where a Pioneer-like anomaly can take a natural place, it is indeed of the first importance to consider these theories with great care because, as stated in the Introduction, the anomaly could be the first hint of a modification of gravity at large scales, with potentially a tremendous impact on galactic and cosmic physics. But if there exist no such theories, the Pioneer anomaly may remain an interesting curiosity with a potentially large impact on navigation in the solar system, but probably lesser importance for fundamental physics.

At this point, it is worth repeating that tests of the equivalence principle (EP) have shown it to be preserved at a very high accuracy level, better than $10^{-12}$ in laboratory experiments as well as in tracking of the motion of Moon on its orbit around the Earth. This is in any case a much higher accuracy than the EP violation which would be needed to account for the Pioneer anomaly : the standard Newton acceleration at $70 \mathrm{UA}$ is of the order 
of $1 \mu \mathrm{m} \mathrm{s}^{-2}$ while the Pioneer anomaly is of the order of $1 \mathrm{~nm} \mathrm{~s}^{-2}$. Should the anomaly be interpreted in terms of an EP violation, the latter would be of the order of $10^{-3}$. This does not contradict the possibility of EP violations which are predicted by unification models 664, 65, 66] and looked for in space experiments with an excellent precision, such as MICROSCOPE [67 and STEP [68. But such violations are expected to occur at a lower level than needed to affect the Pioneer anomaly and we will therefore restrict our attention to a confrontation of GR with alternative metric theories of gravity.

In this well established metric interpretation, the precise form of the coupling between space-time curvature and gravity sources can still be discussed [69]. Like the other fundamental interactions, gravitation may also be treated within the framework of field theory 770, 71, 72. Radiative corrections due to its coupling to other fields then naturally lead to embed GR within a larger class of theories $73,77,75$. Modifications are thus expected to appear 76, 77, 78 in particular, though not only, at large length scales 179, 80, 81, 82. This suggests to consider GR as an effective theory of gravity valid at the length scales for which it has been accurately tested but not necessarily at smaller or larger scales. Note that, in contrast to GR [83], the fourth order theories which are a natural extension of GR show renormalizability as well as asymptotic freedom at high energies 84. This is a strong argument for extending the gravitation theory at scales not already constrained by experiments, for instance using renormalization group trajectories 85. Renormalizability of these theories however comes with a counterpart, that is the problem of ghosts, but it has been argued that this problem does not constitute a definitive deadend for an effective field theory valid in a limited scale domain 86 . In particular, the departure from unitarity is expected to be negligible at ordinary scales tested in present day universe 87.

In the following, we will briefly review the main features of a phenomenological framework which has been recently developed for the purpose of answering the question of the compatibility of the Pioneer anomaly with other gravity tests 21, 22, 23. It will be presented below as covering the whole spectrum of metric extensions of GR which remain in the vicinity of GR. In particular, it will be shown to include as particular cases the PPN extensions as well as the already evoked modifications of Newton force law. Let us stress that this larger family of theories is not just an adhoc extension showing the nice property of letting a place for the Pioneer anomaly. It emerges in a natural manner as the extension of GR induced by radiative corrections due to the coupling of gravity with other fields [88, this idea having been explored before it was noticed that it led to Pioneer-like anomalies 21, 22, 23.

\section{POST-EINSTEINIAN METRIC THEORIES OF GRAVITY}

In order to present the extensions of GR in a simple manner, we start with the linearized version of gravitation theory [21, 22]. We will then present some salient features of the non linear theory [23, 26].

In the linearized treatment, the metric field is represented as a small perturbation $h_{\mu \nu}$ of Minkowski metric $\eta_{\mu \nu}$

$$
g_{\mu \nu}=\eta_{\mu \nu}+h_{\mu \nu} \quad, \quad \eta_{\mu \nu}=\operatorname{diag}(1,-1,-1,-1) \quad, \quad\left|h_{\mu \nu}\right| \ll 7
$$

The field $h_{\mu \nu}$ is a function of position $x$ in spacetime or, equivalently in Fourier space, of wavevector $k$. Gauge invariant observables of the metric theory are given by curvature tensors. In the linearized theory, i.e. at first order in $h_{\mu \nu}$, Riemann, Ricci, scalar and Einstein curvatures have simple expressions in the momentum representation (they are given in Ref. 22]).

These curvature fields are similar to the gauge invariant electromagnetic fields of electrodynamics so that, while being supported by its geometrical interpretation, GR shows essential similarities with other field theories 171, 73. . This suggests that GR may be considered as the low energy effective limit of a more complete unified theory [77, 78] which should describe the coupling of gravity with other fields. In any case, this theory should contain radiative corrections to the graviton propagator, leading to a modification of the Einstein-Hilbert equation (11) and to a momentum dependence of the coupling between curvature and stress tensors. In the weak field approximation, the Einstein tensor, which is divergenceless, has a natural decomposition on the two sectors corresponding to different conformal weights 88, that is also on traceless (conformal weight 0 ) and traced components (conformal weight 1 ).

When considering the isotropic and stationary situation with a point-like and motion-less Sun of mass $M$, the general coupling between curvature and stress tensors is thus described by two running constants $\tilde{G}^{0}$ and $\tilde{G}^{1}$, which depend on the spatial wavevector $\mathbf{k}$ and live in the two sectors (0) and (1). Solutions of the extended gravitation equations (given in Refs. 21, 22]), anew written with spatial isotropic coordinates, depend on two potentials

$$
g_{00}=1+2 \Phi_{N} \quad, \quad g_{r r}=-\left(1-2 \Phi_{N}+2 \Phi_{P}\right)
$$

These two potentials obey Poisson equations with running constants $\tilde{G}_{N}$ and $\tilde{G}_{P}$ given by linear combinations of $\tilde{G}^{0}$ and $\tilde{G}^{1}$

$$
\begin{aligned}
& -\mathbf{k}^{2} \Phi_{a}[\mathbf{k}]=\tilde{G}_{a}[\mathbf{k}] \frac{4 \pi M}{c^{2}} \quad, \quad a=N, P \\
& \tilde{G}_{N} \equiv \frac{4 \tilde{G}^{0}-\tilde{G}^{1}}{3} \quad, \quad \tilde{G}_{P} \equiv \frac{2\left(\tilde{G}^{0}-\tilde{G}^{1}\right)}{3}
\end{aligned}
$$

Standard Einstein equation is recovered when the running constants $\tilde{G}^{0}$ and $\tilde{G}^{1}$ are momentum independent 
and equal to each other, that is also when

$$
\begin{aligned}
& {\left[\tilde{G}_{N}\right]_{\mathrm{st}} \equiv G_{N}, \quad\left[\tilde{G}_{P}\right]_{\mathrm{st}}=0} \\
& {\left[\Phi_{N}(r)\right]_{\mathrm{st}} \equiv \phi(r) \quad, \quad\left[\Phi_{P}(r)\right]_{\mathrm{st}}=0}
\end{aligned}
$$

The two potentials $\Phi_{a}$ will be written as sums of these standard expressions and anomalies which have to remain small

$$
\Phi_{a}(r) \equiv\left[\Phi_{a}(r)\right]_{\mathrm{st}}+\delta \Phi_{a}(r) \quad, \quad\left|\delta \Phi_{a}(r)\right| \ll 1
$$

This linearized form of the extended theory is quite useful for introducing the ideas in terms of an effective field theory of gravitation. It is however not sufficient to deal with the general relation between metric and curvature tensors which involves non linear expressions. It is no more satisfactory for the general discussion of gravity tests as some of them also involve non linearity of the gravitation theory.

It turns out that the extended theory may in fact be given a full non linear formulation, discussed in great details in Ref. 23. Most formulas are thus written more conveniently in terms of Schwartzschild coordinates 89.

$$
\begin{aligned}
& d s^{2}=\bar{g}_{00}(\bar{r}) c^{2} d t^{2}+\bar{g}_{r r}(\bar{r}) d \bar{r}^{2}-\bar{r}^{2}\left(d \theta^{2}+\sin ^{2} \theta d \varphi^{2}\right) \\
& \bar{g}_{\mu \nu}(r) \equiv\left[\bar{g}_{\mu \nu}(r)\right]_{\mathrm{st}}+\delta \bar{g}_{\mu \nu}(r) \quad, \quad\left|\delta \bar{g}_{\mu \nu}(r)\right| \ll 1 \quad(12)
\end{aligned}
$$

with the standard GR solution treated exactly

$$
\left[\bar{g}_{00}\right]_{\mathrm{st}}=1-2 \frac{\kappa}{\bar{r}}=-\frac{1}{\left[\bar{g}_{r r}\right]_{\mathrm{st}}}
$$

and the anomalous metric dealt with at first order. It is possible to define in the non linear theory two potentials $\delta \bar{\Phi}_{N}$ and $\delta \bar{\Phi}_{P}$ which generalize (11) while taking into account the non linear corrections involving powers of $\kappa / \bar{r}$.

We do not reproduce here the corresponding calculations but emphasize a few salient features of the results. First, the phenomenological freedom of the extended framework is represented by the two functions $\delta \bar{g}_{00}(r)$ and $\delta \bar{g}_{r r}(r)$ which contain the same information, through the appropriate transformations [23], as $\delta g_{00}(r)$ and $\delta g_{r r}(r)$, or $\delta \Phi_{N}(r)$ and $\delta \Phi_{P}(r)$, or $\delta G_{N}[\mathbf{k}]$ and $\delta G_{P}[\mathbf{k}]$. They can as well be described by Einstein curvatures $E_{0}^{0}$ and $E_{r}^{r}$ which no longer vanish outside the source [23]. The PPN family is recovered as a particular case which already shows an anomalous behaviour of Einstein curvatures non null apart from the gravity source. Anew, the post-Einsteinian metric theory is nothing but an extension of this anomalous behaviour with more general dependences of the curvatures versus the distance $r$ to the Sun. In loose words, the post-Einsteinian metric theories can be thought of as an extension of PPN metric with $\mathrm{PPN}$ parameters no longer constants but now functions of $r$.

\section{PHENOMENOLOGICAL CONSEQUENCES}

The new phenomenological framework is characterized by the two functions $\delta g_{00}(r)$ and $\delta\left(g_{00} g_{r r}\right)(r)$. The first function represents an anomaly of the Newton potential which has to remain small to preserve the good agreement between GR and gravity tests performed on planetary orbits 53, 54]. Meanwhile, the second sector represents an extension of PPN phenomenology with a scale dependent Eddington parameter $\gamma$. It opens an additional phenomenological freedom with respect to the mere modification of the Newton potential and this freedom opens the possibility to accomodate a Pioneer-like anomaly besides other gravity tests [21, 23].

Recent publications force us to be more specific on the relation between the Pioneer anomaly and modifications of the Newton potential, i.e. anomalies in the first sector according to the terminology of the preceding paragraph. Interpreting the Pioneer anomaly in such a manner requires that $\delta g_{00}$ varies roughly as $r$ at the large radii explored by Pioneer probes. If this dependence also holds at smaller radii 13, or if the anomaly follows a simple Yukawa law [53], one deduces that it cannot have escaped detection in the more constraining tests performed with martian probes [50, 51]. Brownstein and Moffat have explored the possibility that the linear dependence holds at distances explored by Pioneer probes while being cut at the orbital radii of Mars [24]. Other authors [90, 91] have in contrast argued that the ephemeris of outer planets were accurate enough to discard the presence of the required linear dependence in the range of distances explored by the Pioneer probes. This argument has been contested by the authors of Ref. 24] and the conflict remains to be settled.

The authors of Refs. 90, 91 have pushed their claim one step farther by restating their argument as an objection to the very possibility of accounting for the Pioneer anomaly in any viable metric theory of gravity. This claim is clearly untenable because it only considers metric anomalies in the first sector while disregarding those in the second sector. At this point, we want to repeat that the discussion of the compatibility of metric anomalies with observations performed in the solar system has to be done carefully, accounting for the presence of the two sectors as well as for possible scale dependences. This question has already been discussed in Refs. 22, 23] for the cases of deflection experiments on electromagnetic sources passing behind the Sun [39, 40]. It has a particularly critical character for the ranging experiments which involve directly the Shapiro time delay 93 .

The second potential $\delta\left(g_{00} g_{r r}\right)(r)$ naturally produces an anomaly on Doppler tracking of probes with escape trajectories in the outer solar system. This Pioneer-like anomaly can be calculated by taking into account the perturbations on probe motions as well as on light propagation between stations on Earth and probes. The time derivative of the Doppler velocity thus computed can be written as a Doppler acceleration $a$ and the anomaly eval- 
uated as the difference of the values obtained in the extended and standard theories

$$
\delta a \equiv a_{\text {extended }}-a_{\text {standard }} \quad, \quad a \equiv \frac{d v}{d t}
$$

The result of the calculation given in Refs. 21, 22 was unfortunately corrupted by a mistake. The mistake has been corrected in a recent publication Ref. [26] which also contains the evaluation of an annually modulated anomaly coming out, as the secular anomaly, as a natural consequence of the presence of an anomalous metric in the second sector. As observations of such annual anomalies are reported in Ref. [13], this situation certainly pleads for pushing this study farther and comparing the theoretical expectations with the newly recovered Pioneer data [60, 61, 62, 63].

More generally, these data will make available a lot of informations on the status of the probes as well as on the Doppler tracking details, for the whole duration of Pioneer 10 \& 11 missions from their launch to the last data points. The recovery is now completed at JPL 94. and the upcoming data analysis planned as an international effort [95]. Numerous open questions can potentially be solved by this new analysis. The systematics can certainly be much better controlled while several important properties of the force - direction, time variation of the secular anomaly, annual or diurnal modulations, spin contribution, ... - can be more precisely characterized. Then, the availability of early data may make possible to confirm whether or not the anomaly arises at Pioneer 11 at Saturn encounter, as it is suggested by Fig. 7 of Ref. 13. Finally, the data will be confronted to the detailed predictions now available for a variety of theoretical proposals.

If we follow the line of thought presented in this paper, the confrontation of data with extended metric theories of gravitation is of particular interest, as the anomaly observed on the trajectories of the Pioneer $10 \& 11$ probes may well be a first hint of a modification of gravity law in the outer part of the solar system. This possibility would have such a large impact on fundamental physics, astrophysics and cosmology that it certainly deserves further investigations. The evaluations presented in Ref. [26] will allow one to address these questions in a well defined theoretical framework. It is only after a quantitative comparison, taking into account the details known to be important for data analysis [13], that it will be possible to know whether the post-Einsteinian phenomenological framework shows the capability of fitting the Pioneer observations.

When using the corrected expression for the secular anomaly, identification with the observed Pioneer anomaly now points to a quadratic dependence of the second potential with radius. This corresponds to a constant curvature (see the evaluations in Ref. 23]) with an unexpectedly large value in the outer solar system (see Ref. [26]). This quadratic dependence may have to be cut off at distances exceeding the size of the solar system as well as in the inner solar system in order to pass
Shapiro tests on martian probes. As already stressed, it is of crucial importance to check out that the modification of GR needed to produce the Pioneer anomaly does not spoil its agreement with other gravity tests. At the same time, this study can lead to Pioneer-related anomalies, produced by the same metric anomalies, but to be looked for in other kinds of experiments or, in some cases, by having a new look at data of already performed experiments.

The second potential $\delta \Phi_{P}$ has a direct effect on the propagation of light rays. It affects the Eddington deflection experiments as well as the ranging experiments which are sensitive to the Shapiro time delay. These experiments can in fact be described as determining the Eddington parameter $\gamma$, with the new feature that the latter can now depend on the heliocentric distance (more discussions in Ref. 22 for deflections amplified near occultation). The results are reduced to PPN ones when $\gamma$ is a constant. Otherwise, they show that deflection or ranging tests can reveal the presence of $\delta \Phi_{P}$ in the vicinity of the Sun through a space dependence of the parameter $\gamma$. Such a potential dependence might already be looked for through a reanalysis of existing data, such as VLBI measurements [39], Cassini experiment [40], or HIPPARCOS data [96. It may also be studied in the future through higher accuracy Eddington tests, made possible by the global mapping of deflection over the sky in the GAIA project [97], or by the high accuracy LATOR mission [98]. For this kind of tests, the goal can be described as a construction of the dependence of the deflection versus the elongation of the ray with respect to the Sun. This function directly probes the space dependence of the second potential [22] and its unambiguous experimental determination will either produce a clear signature of a deviation of GR or put improved constraints on the existence of the second potential at heliocentric distances smaller than 1 AU.

The presence of $\delta \Phi_{P}$ can also be sensed in planetary tests. In particular, the perihelion precession of planets has been evaluated in the non linear theory 23$]$. The expression there given, written as an anomaly with respect to GR and truncated after leading $\left(\propto e^{0}\right)$ and sub-leading $\left(\propto e^{2}\right)$ orders in the eccentricity $e$ of the planetary orbit, shows that the perihelion precession can be used as a sensitive probe of the value and variation of the second potential. Note that the second potential could in principle be present at the long distances explored by the Pioneer probes, but not at the smaller distance corresponding to the radius of Mars orbit. This entails that it would be extremely interesting to track with accuracy the motions of small bodies which may have significant radial velocities while being at large heliocentric distances. This possibility of testing GR by following small bodies can be considered as a further fundamental challenge for GAIA 97.

Generally speaking, the eccentricity of the orbits plays a key role in Pioneer-related anomalies. It takes large values for Pioneer-like probes which sense $\delta \Phi_{P}$ whereas 
it is zero for circular orbits which do not. This suggests to devote a dedicated analysis to the intermediate situation, not only for the two categories of bound and unbound orbits, but also for the flybies used to bring Pioneer-like probes from the former category to the latter one. It would be worth studying planetary probes on elliptical orbits, for example on transfer orbits from Earth to Mars or Jupiter. Another natural target for such a study could be LISA with its three crafts on slightly elliptical orbits 99 .

Finally, there are strong motivations for new missions designed to study the anomaly and try to understand its origin 100. A cheaper and quicker alternative could be to fly dedicated passenger instruments on planetary missions with different primary purposes. In the meantime, a wise strategy is to develop and validate enabling tech- nologies, such as laser and radio techniques for ranging, accelerometers for controlling the deviation from geodesic motion, accurate clocks on board for measuring separately the two components of the metric.

\section{Acknowledgements}

Thanks are due for discussions to the members of the "Deep Space Gravity Probe" team (H. Dittus et al) [100, of the "Pioneer Anomaly Investigation Team" (S.G. Turushev et al) 95, and of the "Groupe Anomalie Pioneer", in particular F. Bondu, P. Bouyer, B. Christophe, J.-M. Courty, B. Foulon, S. Léon, A. Lévy, G. Metris and P. Touboul.
[1] C.M. Will, Theory and experiment in gravitational physics (Cambridge U. P., Cambridge, 1993).

[2] C.M. Will, Living Rev. Rel. 44 (2001); http://relativity.livingreviews.org/.

[3] E. Fischbach and C. Talmadge, The Search for Non Newtonian Gravity (Springer Verlag, Berlin, 1998).

[4] E.G. Adelberger, B.R. Heckel and A.E. Nelson, Ann. Rev. Nucl. Part. Sci. 5377 (2003).

[5] A. Aguirre, C.P. Burgess, A. Friedland et al , Class. Quantum Grav. 18 R223 (2001).

[6] A.G. Riess, A.V. Filippenko, P. Challis et al, Astron. J. 1161009 (1998).

[7] S. Perlmutter, G. Aldering, G. Goldhaber et al, Ap. J. 517565 (1999).

[8] S. Perlmutter, M.S. Turner and M. White, Phys. Rev. Lett. 83670 (1999).

[9] R.H. Sanders and S.S. McGaugh, Annu. Rev. Astron. Astrophys. 40263 (2002).

[10] A. Lue, R. Scoccimari and G. Starkman, Phys. Rev. D69 044005 (2004).

[11] S.M. Carroll, V. Duvvuri, M. Trodden et al, Phys. Rev. D70 043528 (2004).

[12] J.D. Anderson, P.A. Laing, E.L. Lau et al, Phys. Rev. Lett. 812858 (1998).

[13] J.D. Anderson, P.A. Laing, E.L. Lau et al, Phys. Rev. D65 082004 (2002).

[14] J.D. Anderson, M.M. Nieto and S.G. Turyshev, Int. J. Mod. Phys. D11 1545 (2002).

[15] J.D. Anderson, E.L. Lau, S.G. Turyshev et al, Mod. Phys. Lett. A17 875 (2003).

[16] M.M. Nieto and S.G. Turyshev, Class. Quantum Grav. 214005 (2004).

[17] S.G. Turyshev, M.M. Nieto and J.D. Anderson, grqc/0409117.

[18] M.M. Nieto, Phys. Rev. D72 083004 (2005).

[19] M.M. Nieto, S.G. Turyshev and J.D. Anderson, Phys. Lett. B613 11 (2005).

[20] O. Bertolami and J. Paramos, Class. Quantum Grav. 213309 (2004).

[21] M.-T. Jaekel and S. Reynaud, Mod. Phys. Lett. A20 1047 (2005).

[22] M.-T. Jaekel and S. Reynaud, Class. Quantum Grav.
222135 (2005).

[23] M.-T. Jaekel and S. Reynaud, Class. Quantum Grav. 23777 (2006).

[24] J.R. Brownstein and J.W. Moffat, Class. Quantum Grav. 233427 (2006).

[25] C. Lämmerzahl, O. Preuss and H. Dittus, in Lasers, Clocks, and Drag-Free: Technologies for Future Exploration in Space and Tests of Gravity (Springer, Berlin, 2006) p.75.

[26] M.-T. Jaekel and S. Reynaud, gr-qc/0610155, to appear in Class. Quantum Grav..

[27] A. Einstein, Jahrbuch der Radioaktivität und Elektronik 4411 (1907).

[28] A. Einstein, Annalen der Physik 35898 (1911).

[29] J.G. Williams, X.X. Newhall and J.O. Dickey, Phys. Rev. D53 6730 (1996).

[30] R.W. Hellings, P.J. Adams, J.D. Anderson et al, Phys. Rev. Lett. 511609 (1983).

[31] A. Einstein, Sitz. der Preuss. Akad. der Wissenschaften zu Berlin 844 (1915).

[32] D. Hilbert, Nachr. von der Gesellshaft der Wissenshaften zu Göttingen 395 (1915).

[33] A. Einstein, Annalen der Physik 49769 (1916).

[34] A.S. Eddington, The mathematical theory of relativity (Cambridge U. P., Cambridge, 1957).

[35] H.P. Robertson in Space age astronomy (Academic Press, , 1962).

[36] D.H. Ross and L.I. Schiff, Phys. Rev. 1411215 (1966).

[37] K. Nordtvedt, Phys. Rev. 1691014 \& 1017 (1968).

[38] C.M. Will and K. Nordtvedt, Ap. J. 177757 (1972); K. Nordtvedt and C.M. Will, ibidem 775.

[39] S.S. Shapiro, J.L. Davis, D.E. Lebach et al, Phys. Rev. Lett. 92121101 (2004).

[40] B. Bertotti, L. Iess and P. Tortora, Nature 425374 (2003).

[41] C. Talmadge et al, Phys. Rev. Lett. 611159 (1988).

[42] K. Nordtvedt, gr-qc/0301024.

[43] C.D. Hoyle et al, Phys. Rev. Lett. 861418 (2001); Phys. Rev. D70 042004 (2004).

[44] J. Long et al , Nature 421922 (2003).

[45] J. Chiaverini et al, Phys. Rev. Lett. 90151101 (2003).

[46] M. Bordag, U. Mohideen and V.M. Mostepanenko, 
Phys. Rep. 3531 (2001).

[47] A. Lambrecht and S. Reynaud, in Poincaré Seminar 2002, eds. B. Duplantier and V. Rivasseau (Birkhaüser Verlag, Basel, 2003), p. 109.

[48] R.S. Decca et al , Phys. Rev. D68 116003 (2003).

[49] F. Chen et al, Phys. Rev. A69 022117 (2004).

[50] R.D. Reasenberg et al, Ap. J. 234 L219 (1979).

[51] J.D. Anderson, M. Gross, K. Nordtvedt et al, Ap. J. 459365 (1996).

[52] N.I. Kolosnitsyn and V.N. Melnikov, Gen. Rel. Grav. 361619 (2004).

[53] M.-T. Jaekel and S. Reynaud, Int. J. Mod. Phys. A20 2294 (2005).

[54] J. Coy, E. Fischbach, R. Hellings et al, private communication (2003).

[55] R.O. Fimmel, W. Swindell and E. Burgess, NASA Publication SP-349/396 (NASA, Washington, 1977); electronic version at http://history.nasa.gov/SP349/sp349.htm.

[56] R.O. Fimmel, J.A. Van Allen and E. Burgess, NASA Publication SP-446 (NASA, Washington, 1980).

[57] S.W. Asmar, J.W. Armstrong, L. Iess et al, Radio Science 40 RS2001 (2005).

[58] T.D. Moyer, Formulation for Observed and Computed Values of Deep Space Network Data Types for Navigation (Wiley, New York, 2003); http://descanso.jpl.nasa.gov/.

[59] C. Markwardt, gr-qc/0208046; http://lheawww.gsfc.nasa.gov/users/craigm/atdf/.

[60] S.G. Turyshev, M.M. Nieto and J.D. Anderson, EAS Publ.Ser. 20243 (2006).

[61] M.M. Nieto and J.D. Anderson, Class. Quantum Grav. 225343 (2005).

[62] S.G. Turyshev, V.T. Toth, L.R. Kellogg et al , Int. Journ. Mod. Phys. D15 1 (2006).

[63] V.T. Toth and S.G. Turyshev, gr-qc/0603016.

[64] T. Damour, Class. Quantum Grav. 13 A33 (1996).

[65] T. Damour, F. Piazza and G. Veneziano, Phys. Rev. D66 046007 (2002).

[66] J.M. Overduin, Phys. Rev. D62 102001 (2000).

67] MICROSCOPE Site @ CNES http://smsc.cnes.fr/MICROSCOPE.

[68] STEP Site @ Stanford Univ. http://einstein.stanford.edu/STEP/.

[69] S. Weinberg, Gravitation and Cosmology (Wyley, New York, 1972)

[70] W.E. Thirring, Ann. of Phys. 1696 (1961).

[71] R.P. Feynman, Acta Phys. Polonica 24711 (1963).

[72] S. Weinberg, Phys. Rev. 138 B988 (1965).
[73] R. Utiyama and B. De Witt, J. Math. Phys. 3608 (1962).

[74] S. Deser and P. van Nieuwenhuizen, Phys. Rev. D10 401 (1974).

[75] D.M. Capper, M.J. Duff and L. Halpern, Phys. Rev. D10 461 (1974).

[76] K.S. Stelle, Phys. Rev. D 16 (1977) 953; Gen. Rel. Grav. 9353 (1978).

[77] A.D. Sakharov, Dokl. Akad. Nauk SSSR 17770 (1967).

[78] R.J. Adler, Rev. Mod. Phys. 54729 (1982).

[79] T. Goldman, J. Pérez-Mercader, F. Cooper et al, Phys. Lett. B281 219 ().

[80] C. Deffayet, G. Dvali, G. Gabadadze et al, Phys. Rev. D65 044026 (2002).

[81] G. Dvali, A. Gruzinov, M. Zaldarriaga, Phys. Rev. D68 024012 (2003).

[82] G. Gabadadze and M. Shifman, Phys. Rev. D69 124032 (2004).

[83] G. t'Hooft and M. Veltman, Ann. Inst. H. Poincaré A20 69 (1974).

[84] E.S. Fradkin and A.A. Tseytlin, Nucl. Phys. B201 469 (1982).

[85] O. Lauscher and M. Reuter, Class. Quantum Grav. 19 483 (2002).

[86] J.Z. Simon, Phys. Rev. D41 3720 (1990).

[87] S.W. Hawking and T. Hertog, Phys. Rev. D65 103515 (2002).

[88] M.-T. Jaekel and S. Reynaud, Annalen der Physik 468 (1995).

[89] C.W. Misner, K.S. Thorne and J.A. Wheeler, Gravitation (Freeman, New York, 1972).

[90] L. Iorio and G. Giudice, New Astron. 11600 (2006).

[91] K. Tangen, gr-qc/0602089.

[92] J.W. Moffat, Class. Quantum Grav. 236767 (2006).

[93] I.I. Shapiro, Rev. Mod. Phys. 71 S41 (1999).

[94] Contact: Slava G. Turyshev ituryshev@jpl.nasa.govi.

[95] See the pages of the Pioneer Anomaly Investigation Team at the International Space Sciences Institute http://www.issi.unibe.ch/teams/Pioneer/.

[96] HIPPARCOS Site @ ESA http://www.rssd.esa.int/Hipparcos.

[97] GAIA Site @ ESA http://www.rssd.esa.int/Gaia.

[98] The LATOR Collaboration: S.G. Turyshev et al , grqc/0506104

[99] LISA Site @ ESA http://www.rssd.esa.int/Lisa.

[100] The Pioneer Explorer Collaboration: H. Dittus et al, gr-qc/0506139. 